\title{
Empleo de agua proveniente del lavado de camiones mixers en la producción de hormigones sustentables: Evaluación de la trabajabilidad y resistencia
}

\author{
C.G. Caballero ${ }^{1}$, A.J. Palacio ${ }^{1}$, A. Alzogaray ${ }^{1}$, F.A. Avid ${ }^{1}$, J.D. Sota ${ }^{1 *}$. \\ *Autor de Contacto: jdsota@gmail.com \\ ${ }^{1}$ Grupo GIICMA, Universidad Tecnológica Nacional UTN-Facultad Regional Concordia, Concordia, Entre Ríos, \\ Argentina.
}

\section{RESUMEN}

En muchos países, las plantas elaboradoras de hormigón emplean camiones moto hormigoneros para su producción y en el proceso usan gran cantidad de agua potable. Este consumo se da en la producción de hormigón, pero además se debe considerar el agua utilizada para lavar los camiones mixers, ya que esto también genera una cantidad considerable de agua residual que no se puede eliminar sin un tratamiento previo. Por lo tanto, el objetivo de este trabajo es estudiar el empleo de agua reciclada, producto del lavado de camiones mixers en la producción de hormigón, reduciendo el consumo de agua potable. Se desarrollan tres dosificaciones, una de referencia producida con agua potable, una dosificación con $25 \%$ de remplazo por agua residual y una tercera con un $35 \%$ de reemplazo. Para el análisis de los hormigones, se comprobaron sus propiedades en estado fresco y endurecido, evaluando la trabajabilidad mediante el ensayo de asentamiento y su resistencia a la compresión a los 7, 28 y 56 días. Los resultados mostraron que el hormigón producido con estos porcentajes de reemplazo presentó la misma resistencia a la compresión que el hormigón patrón, y en estado fresco se observa un incremento de la fluidez con el aumento del porcentaje de reemplazo.

Palabras clave: Agua reciclada; hormigón sustentable; producción de hormigón; reúso; materiales eco-eficientes.

\section{ABSTRACT}


In many countries, concrete plants use mixer trucks for their production and this process consumes a large quantity of drinking water. In addition to the production of concrete, the water used to wash the mixer trucks should also be considered, since this also generates a considerable amount of residual water that cannot be disposed of without prior treatment. Therefore, the objective of this study is to reuse the waste water generated by the washing of mixer trucks in the production of concrete, thus avoiding the consumption of drinking water. Three compositions were developed, a reference composition produced with drinking water, a composition with $25 \%$ replacement by waste water and a composition with a $35 \%$ replacement. For the analysis of the concretes, its properties were checked in the fresh and hardened state, assessing the workability by means of the concrete slump test and their resistance to compression at 7,28 and 56 days. The results show that the operation produced with these replacement percentages presented the same resistance to compression as the standard concrete, and in the fresh state an increase in fluidity is observed with the increase in the replacement percentage.

Palabras clave: recycled water; sustainable concrete; concrete production; reuse; eco-efficient materials.

\section{RESUMO}

Em muitos países, as fábricas de concreto usam caminhões betoneira para sua produção e consomem uma grande quantidade de água potável no processo. Esse consumo ocorre na produção de concreto, mas a água utilizada na lavagem dos caminhões betoneira também deve ser considerada, pois gera uma quantidade considerável de água residual que não pode ser eliminada sem tratamento prévio. Portanto, o objetivo deste trabalho é estudar a utilização da água reciclada, produto da lavagem de caminhões betoneiras na produção de concreto, reduzindo o consumo de água potável. São desenvolvidas três dosagens, uma de referência produzida com água potável, uma dosagem com substituição de $25 \%$ por água residual e uma terceira com substituição de $35 \%$. Para a análise dos concretos, foram verificadas suas propriedades no estado fresco e endurecido, avaliando-se a trabalhabilidade por meio do ensaio de abatimento e sua resistência à compressão aos 7, 28 e 56 dias. Os resultados mostram que o desempenho produzido com esses percentuais de substituição apresentou a mesma resistência à compressão do concreto padrão, sendo que no estado fresco observa-se um incremento da fluidez com o aumento do percentual de substituição.

Palavras clave: água reciclada; concreto sustentável; produção de concreto; reuso; materiais eco eficientes. 


\section{INTRODUCCIÓN}

En la Argentina y en las regiones industriales ubicadas en general en las provincias tales como Entre Ríos, Santa Fe, Buenos Aires, Córdoba, es claro y evidente la necesidad de diseñar e implementar sistemas de tratamiento de las aguas residuales en la industria del hormigón elaborado. Esto es así debido a la falta de aplicación de los mismos o, en algunos casos, el tratamiento precario para las operaciones de lavado de planta y camiones mixers luego del transporte de material. Es por esto que las aplicaciones de sistemas de tratamientos de aguas residuales constituyen una alternativa válida para la reducción del impacto ambiental y la optimización de los recursos naturales utilizados en la producción de hormigón.

Sin embargo, se debe tener en cuenta que las propiedades de las aguas residuales industriales y específicamente las de la industria del hormigón, dependen en su mayoría de la composición química, factor que resulta importante a la hora de tomar decisiones respecto a su potencial reutilización. También es importante tener en cuenta que las aguas residuales y los agregados que puedan recuperarse en los diferentes pasos del procesamiento de un sistema de tratamiento varían en sus características fisicoquímicas (Ricardo de Matos et.al., 2020). Por lo tanto, no solo se necesita una base de datos actualizada respecto a los resultados y valores admisibles de los análisis cuantitativos y cualitativos de las aguas residuales, sino también la elaboración de manuales y reglamentaciones que regulen las prácticas actuales de eliminación de los desechos en dicha industria (Balzamo et.al., 2012). Tal es así que se requieren más investigaciones para encontrar un sistema de tratamiento que genere una fuente estable y confiable de agua reutilizable, con una variabilidad de composición físico-química mínima para una utilización óptima. Por consiguiente, el tratamiento y la reutilización del agua de desecho conforma hoy en día objeto de varias investigaciones, convirtiéndose en una problemática ambiental a resolver y estudiar muy importante.

En la industria de la construcción a pesar del notable avance y desarrollo de nuevos materiales, tecnologías y metodologías de construcción, el hormigón continúa siendo el material más utilizado, especialmente en lo que respecta a la construcción de viviendas, con el fin de responder a la demanda de la población creciente. Pero el alto consumo de agua y la generación de aguas residuales sumado a la gestión de estos factores en la industria del hormigón elaborado, se han convertido en cuestiones ambientales muy importantes a resolver y tratar.

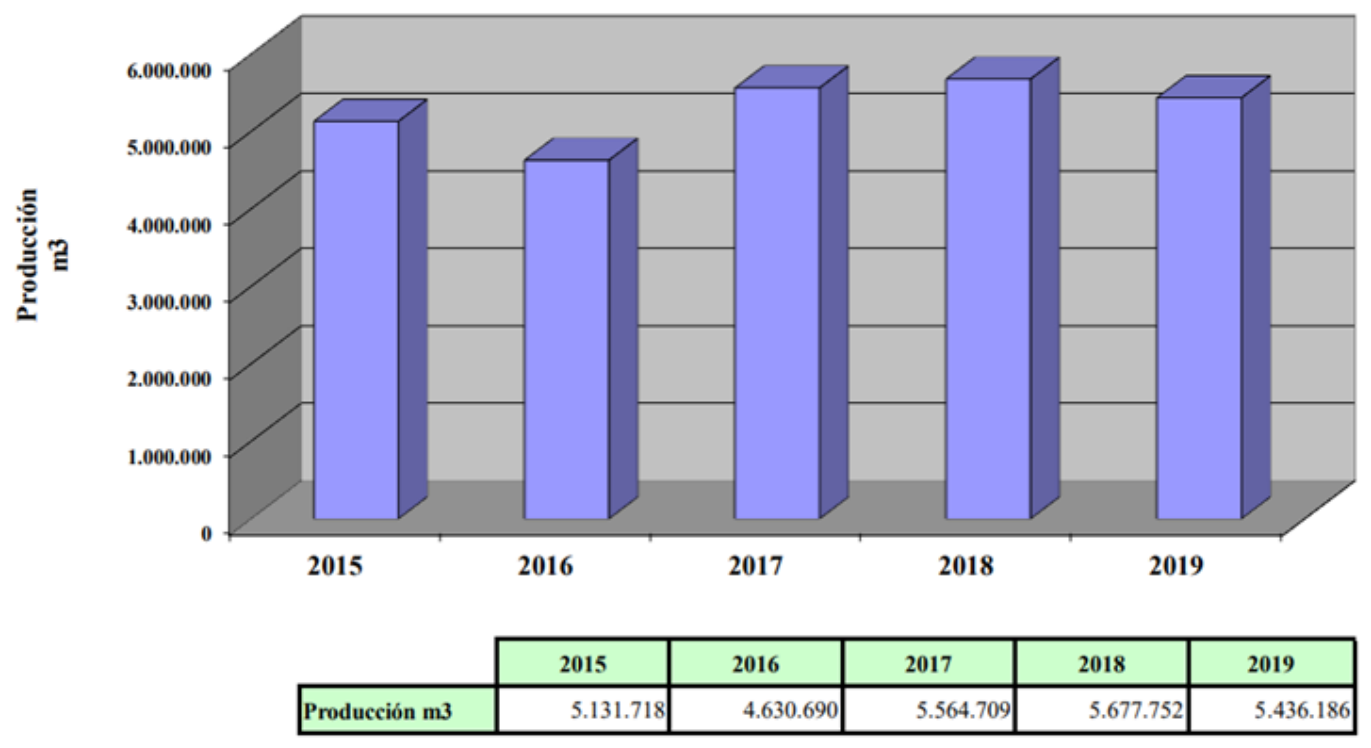

Figura 1. Producción anual de empresas elaboradoras de hormigón asociadas a la AAHE. (AAHE, 2019). 
En Argentina, un índice indicativo de las cantidades de hormigón que se producen, como así también de la actividad del sector, es el consumo de cemento, registrándose en el año 2019 (último año completo disponible), un consumo total de 11.003 .359 toneladas de cemento, o su equivalente valor per-cápita de 245 kilogramos de cemento por habitante (AFCP, 2019). Alrededor de 5.436.186 de metros cúbicos de hormigón se producen anualmente en plantas elaboradoras (Figura 1), de las cuales tan solo un $8 \%$ cumplen con certificación de gestión de calidad ISO 9001:2015 y un $21 \%$ elaboran bajo estándares de calidad y servicio aceptables, el 71 \% restante no cumplen y no califican con los estándares de calidad en la producción y el ambiente, lo que da una idea de la gestión actual de los residuos de la industria (Segerer, 2020).

Se estima que, al final de cada jornada, queda un residuo de aproximadamente $300 \mathrm{~kg}$ de hormigón plástico en cada camión mezclador (Sandrolini y Franzoni, 2001). Dicho sobrante si bien puede reutilizarse mediante el empleo de aditivos estabilizadores de hidratación, lo habitual es que sea lavado, debido a los costos que conllevan los aditivos mencionados, "a priori" más costosos en comparación con el uso de agua. Cabe mencionar además que, en las plantas elaboradoras locales, dichos lavados se realizan sin reservas ni regulación alguna en lo que respecta al consumo, vertido y/o devolución al ambiente del agua interviniente.

\section{MATERIALES Y PROCEDIMIENTO}

Como parte de una primera etapa de investigación, con la colaboración de una empresa local de la ciudad de Concordia en la provincia de Entre Ríos, Argentina, se procedió a elaborar pastones de prueba. Para los mismos se contó con los materiales de acopio tales como agregados gruesos y finos, cemento y aditivos, como así también con la dosificación de un hormigón de línea de la empresa, la cual opera de acuerdo con CIRSOC 201/2005 bajo el modo de producción MODO I, a través de un sistema de gestión de la calidad, con procesos de producción basados en la norma ISO 9001:2015. De esta manera se buscó evaluar, en una primera instancia, todas aquellas variaciones que puedan encontrarse respecto al hormigón de referencia, para posteriormente definir el correcto marco teórico de aplicación del agua reciclada.

\subsection{Cemento}

Para este trabajo se utilizó un cemento portland compuesto (CPC 50) según Norma IRAM 50000 - "Cementos de uso general", el cual fue extraído de silos de acopio de planta elaboradora. Este tipo de cemento es el de uso comercial habitual en el país, especialmente en la Región Centro y Mesopotamia. Su composición es presentada en la Tabla 1.

Tabla 1. Tipo de cemento y composición según Norma IRAM 50000.

\begin{tabular}{|c|c|c|c|c|c|c|c|}
\hline \multicolumn{3}{|c|}{ Designación } & \multicolumn{5}{|c|}{ Composición (g/100 g) } \\
\hline $\begin{array}{l}\text { Tipo de } \\
\text { Cemento }\end{array}$ & $\begin{array}{l}\text { Nomen } \\
\text { clatura }\end{array}$ & $\begin{array}{c}\text { Clase } \\
\text { Resistente }\end{array}$ & $\begin{array}{c}\text { Clinker } \\
+ \\
\text { sulfato } \\
\text { de } \\
\text { calcio }\end{array}$ & $\begin{array}{c}\text { Puzolana o } \\
\text { ceniza } \\
\text { volante } \\
\text { silícea } \\
(P \text { o } C V) \\
\end{array}$ & $\begin{array}{l}\text { Escoria } \\
\text { (E) }\end{array}$ & $\begin{array}{c}\text { "Filler" } \\
\text { calcáreo } \\
\text { (F) }\end{array}$ & $\begin{array}{l}\text { Compuestos } \\
\text { minoritarios }\end{array}$ \\
\hline $\begin{array}{l}\text { Cemento } \\
\text { Portland } \\
\text { Compuesto }\end{array}$ & $\mathrm{CPC}$ & 50 & $94-65$ & \multicolumn{3}{|c|}{$\begin{array}{c}\text { Dos o más, con } 6 \leq(\mathrm{P}+\mathrm{E}+\mathrm{F}) \leq \\
35 \text { y } \mathrm{F} \leq 35\end{array}$} & $0-5$ \\
\hline
\end{tabular}




\subsection{Agregado Grueso}

Se utilizó un agregado natural silíceo tipo canto rodado, el cual es extraído de canteras ubicadas en la región Mesopotámica de la Argentina, específicamente en la provincia de Entre Ríos y Corrientes, con un tamaño máximo nominal de agregado 6 a $20 \mathrm{~mm}$, módulo de finura de 6,73 y una curva de distribución granulométrica indicada en la Figura 2, de acuerdo con los límites de la Norma IRAM 1627 para agregados gruesos.

\subsection{Agregado Fino}

Se utilizó una arena natural gruesa tipo silícea de lavado, la cual es extraída de canteras ubicadas en las márgenes del Río Uruguay, en la provincia de Entre Ríos, módulo de finura de 2,61 y una distribución granulométrica indicada en la Figura 3, de acuerdo a los límites de la Norma IRAM 1627 para agregados finos.

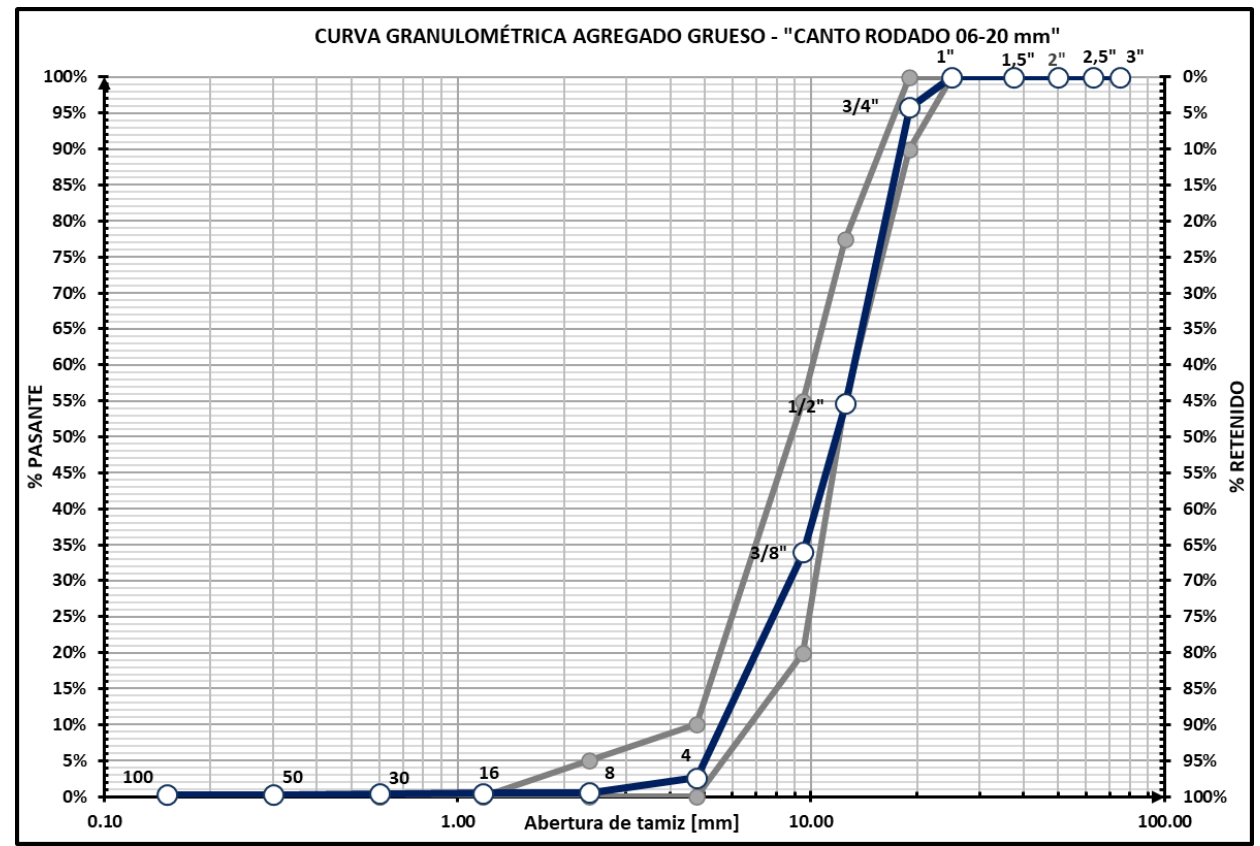

Figura 2. Granulometría "canto rodado" 06-20 [mm].

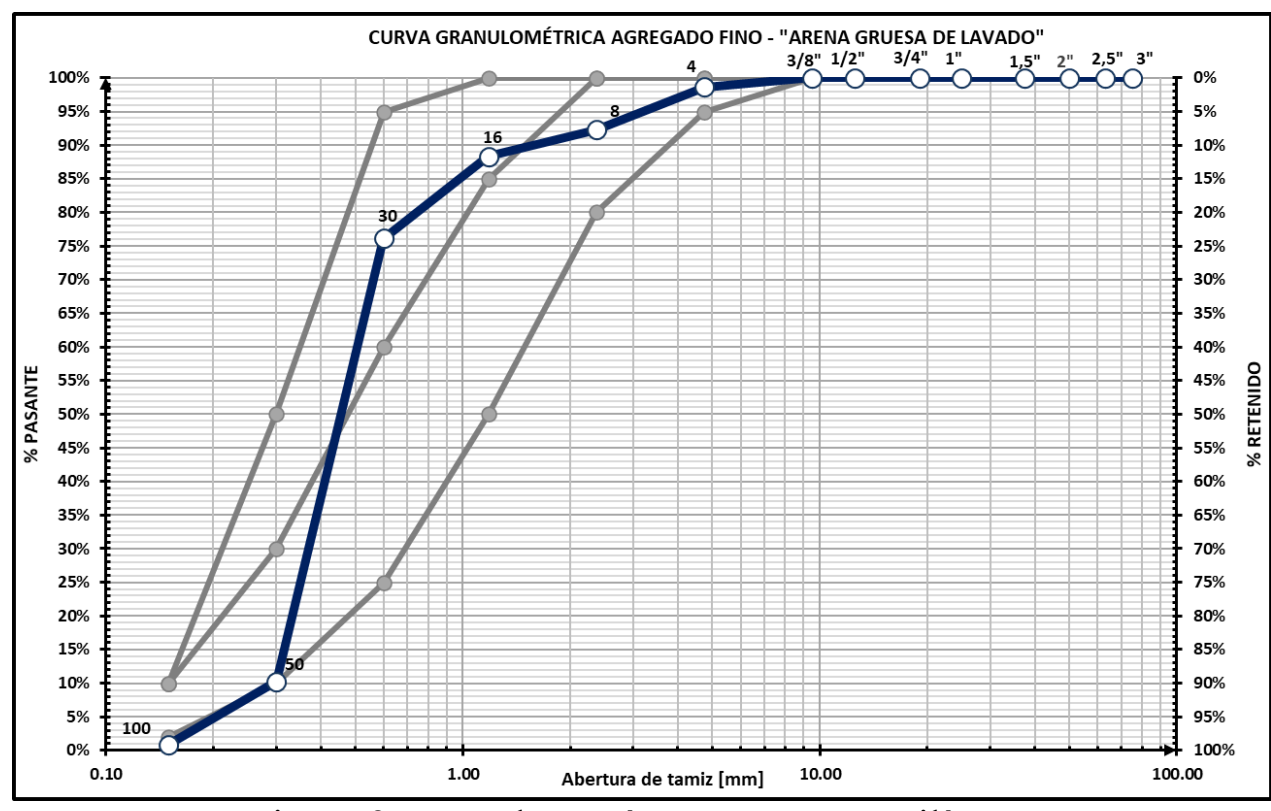

Figura 3. Granulometría "arena gruesa silícea". 


\subsection{Aditivo Químico}

En este caso se utilizó un aditivo plastificante y superfluidificante de medio rango, de uso comercial en la Argentina, cuya composición química se indica en la Tabla 2.

Tabla 2. Composición química aditivo plastificante y superfluidificante.

\begin{tabular}{|c|c|}
\hline Nombre químico & Concentración (\%) \\
\hline Naphtalensulfonic acid-formaldehyde condensate sodium salt & $\geq 5-<10$ \\
\hline 1,2-bencisotiazol-3(2H)-ona & $\geq 0,1-<1$ \\
\hline
\end{tabular}

\subsection{Agua Reciclada}

El agua reciclada se obtuvo de la misma empresa, la cual cuenta con un sistema de piletas de sedimentación y decantación, mediante el cual se realiza el lavado diario de los camiones mixers. De estas piletas se extrajeron diferentes muestras de agua, las que luego fueron utilizadas para la elaboración de los pastones de prueba. El sistema de tratamiento consta de un total de 5 piletas continuas en las que el intercambio se realiza mediante el trasvase por gravedad. Puntualmente se extrajeron muestras de las piletas $\mathrm{N}^{\circ} 1$ y $\mathrm{N}^{\circ} 4$ del sistema, correspondiendo la primera a la pileta donde se vuelcan directamente los residuos del camión y de lavado, y la segunda mencionada corresponde a la pileta final del sistema, previo al almacenamiento y filtrado. En la Figura 4 se puede observar la disposición de las piletas y el sentido del escurrimiento del agua de lavado.

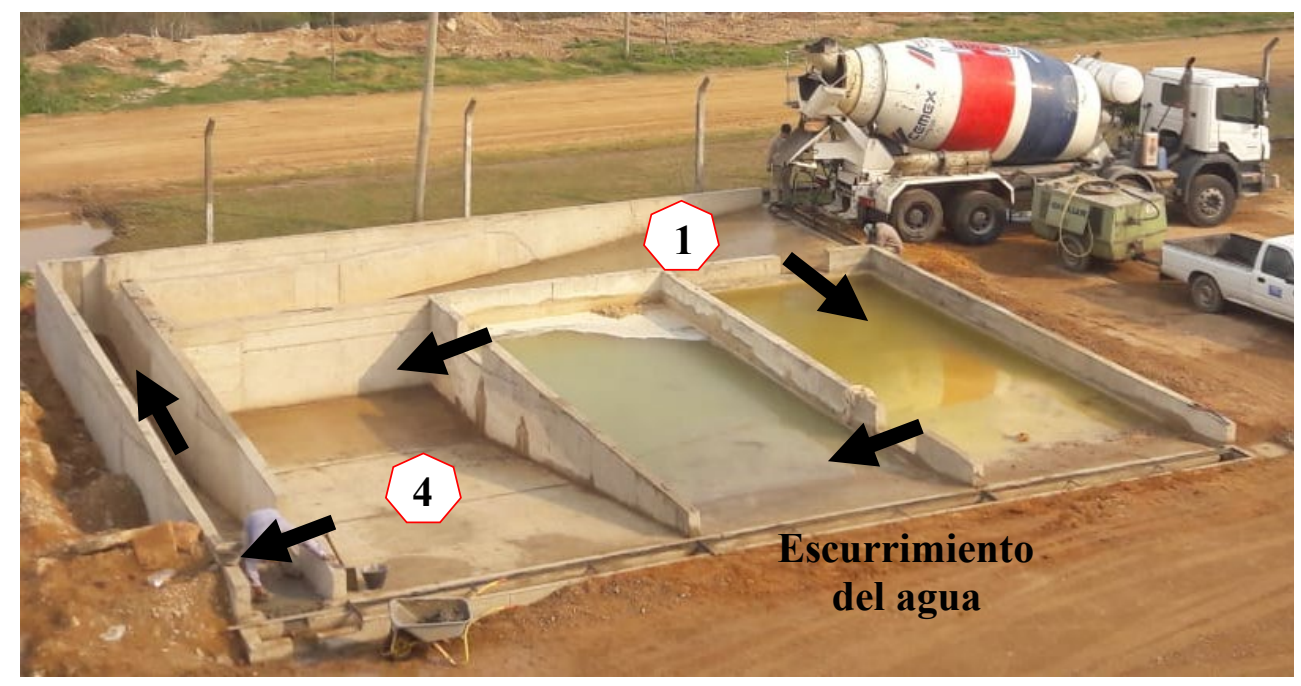

Figura 4. Sistema de piletas de sedimentación y decantación de sólidos de agua de lavado de camiones mixers.

Se recolectaron 60 litros de agua proveniente del lavado de los camiones, la cual fue almacenada en bidones y botellas para el desarrollo de las pruebas de laboratorio. El agua recogida presenta cierto grado de turbidez por las partículas sólidas en suspensión.

De las muestras extraídas de las piletas 1 y 4 se realizó la evaluación de temperatura, nivel de pH, conductividad y contenido de sales disueltas, utilizando un medidor multiparamétrico. En las Figuras 5 y 6 obtenidas a partir del análisis de los datos de las muestras de agua, se observa una significativa alcalinidad del agua de la Pileta $\mathrm{N}^{\circ} 1$ (la cual recibe de manera directa la descarga del agua reciclada, mezclada con los sobrantes de hormigón). Esta presenta, a su vez, un valor promedio de $\mathrm{pH}$ de $11,58 \mathrm{y}$ el contenido total de sales disueltas o TDS posee un valor promedio de 3010,83 ppm. En cambio, los valores promedios de la Pileta $\mathrm{N}^{\circ} 4$ (la cual se encuentra al final del sistema de piletas, ver Figura 4) son respectivamente 10,78 para el $\mathrm{pH}$ y 744,33 ppm para el 
TDS. Claramente se demuestra la mayor concentración de sales disueltas y la alta alcalinidad que posee el agua de reciclado de los camiones mixers sin tratamiento previo. Incluso si solo se la somete a una decantación o sedimentación, se reducen considerablemente dichos valores.

A partir de dichos resultados, se decidió elaborar los pastones de prueba utilizando el agua reciclada de la Pileta $\mathrm{N}^{\circ} 4$, considerando las significativas variaciones en los valores representativos de $\mathrm{pH}$ y sales disueltas respecto a los valores límites de 4 para $\mathrm{pH}$ y de 50000 ppm para TDS, según Norma IRAM 1601 (IRAM 1601, 2012).

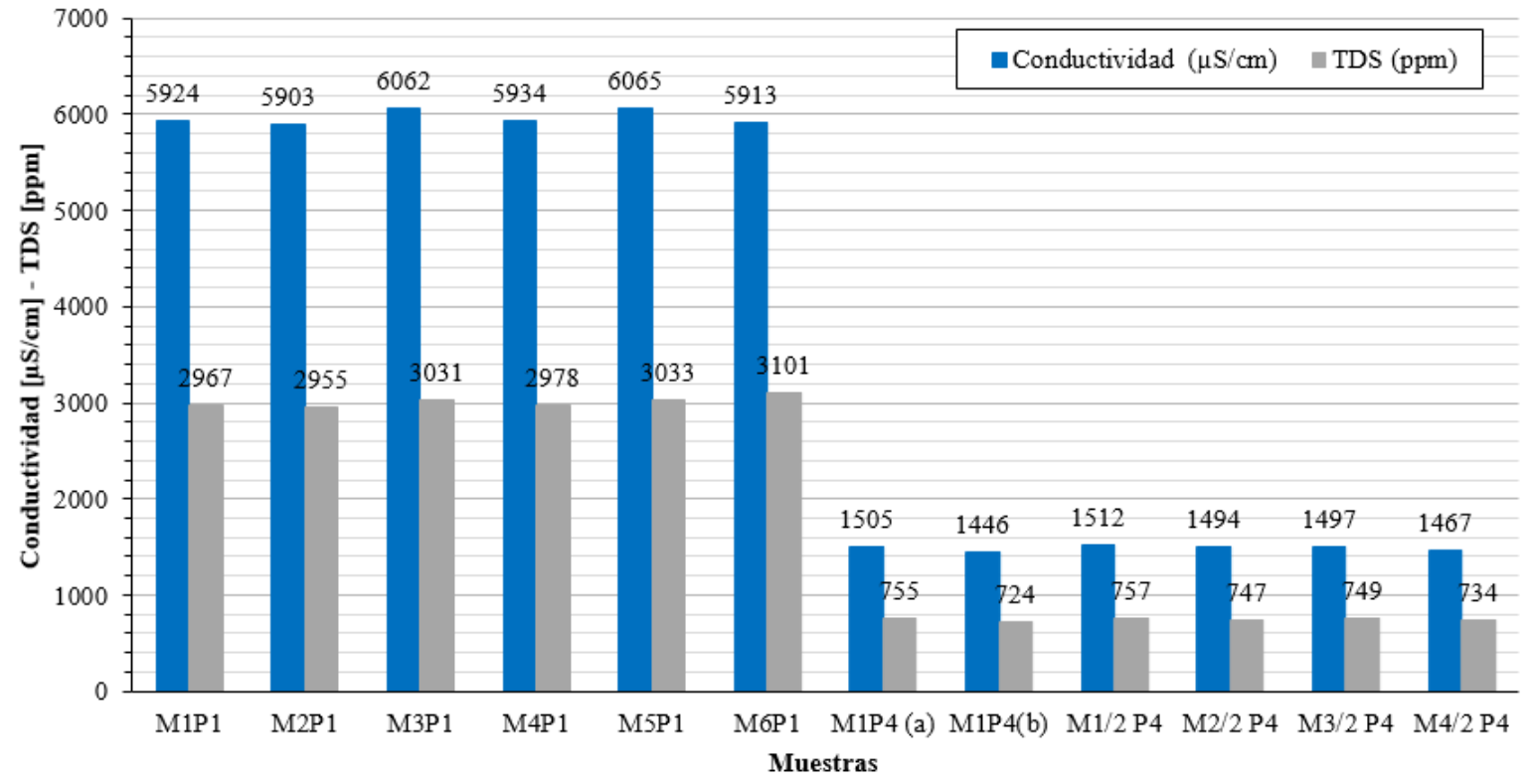

Figura 5. Conductividad y contenido total de sales disueltas. Pileta 1 vs Pileta 4.

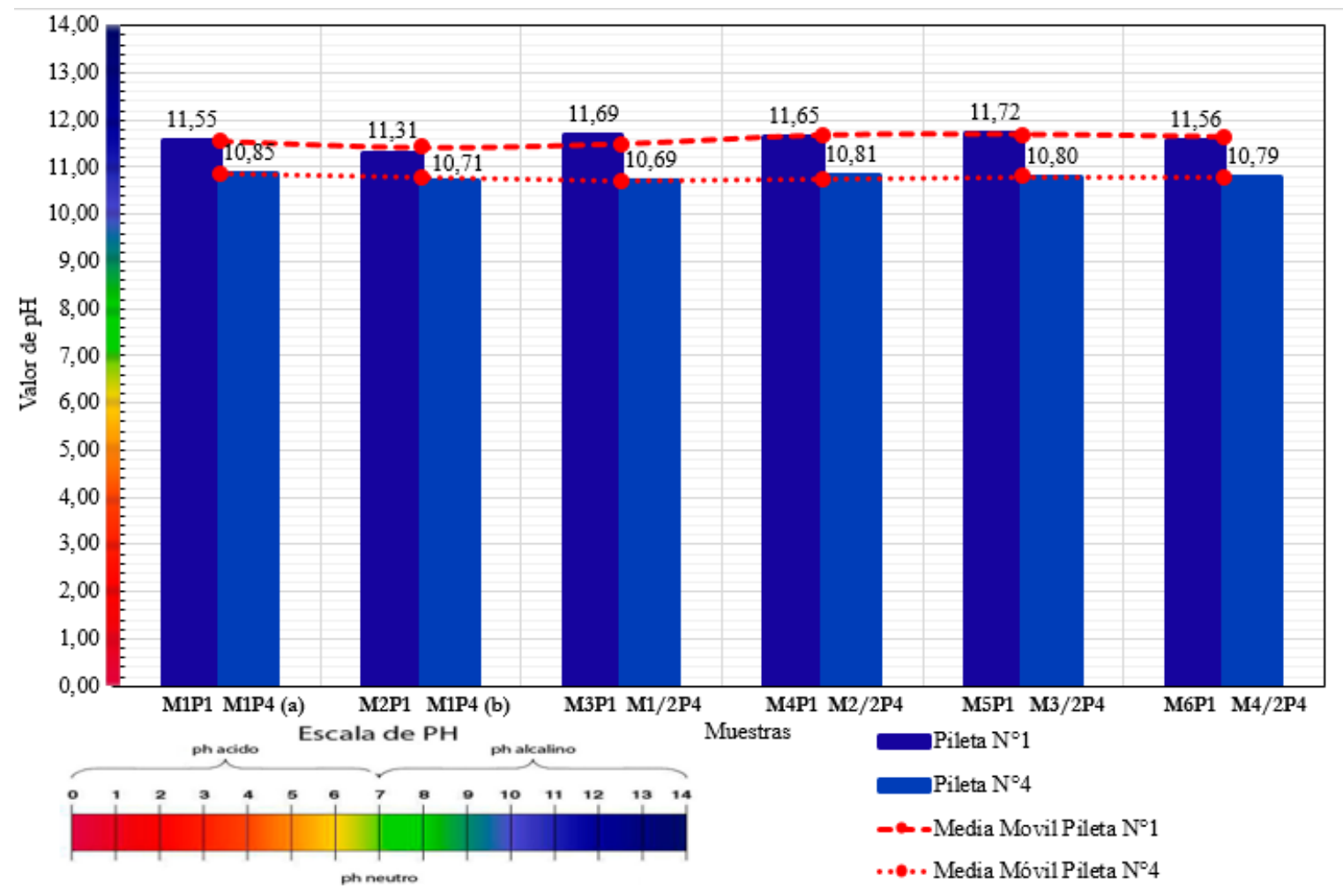

Figura 6. Valores de $\mathrm{pH}$ agua reciclada Pileta 1 vs Pileta 4. 


\subsection{Dosificación}

El diseño de la mezcla fue seleccionado en base a una dosificación tradicional de la empresa elaboradora. En este caso se optó por un hormigón clase H30 (INTI-CIRSOC, 2005) el cual es de producción y uso habitual en la región. En la Tabla 3 se puede observar esta dosificación, la cual corresponde al pastón de referencia o base. A su vez, esta dosificación se replicó en los pastones $\mathrm{N}^{\mathrm{o}} 2$ y $\mathrm{N}^{\mathrm{o}} 3$, pero con el reemplazo parcial del agua potable por agua proveniente del lavado de los camiones mixers. Este reemplazo se realizó en porcentajes del $25 \%$ y $35 \%$ respectivamente.

Tabla 3. Dosificación pastón de prueba $N^{\circ} 01-0 \%$ Agua Reciclada.

\begin{tabular}{|c|c|c|c|c|c|c|c|}
\hline \multirow{2}{*}{ Material } & \multirow{2}{*}{$\begin{array}{c}\text { Densidad } \\
\text { Kg/dm3 }\end{array}$} & $\begin{array}{c}\text { Peso } \\
\mathbf{K g}\end{array}$ & $\begin{array}{c}\text { Volumen } \\
\mathbf{d m 3}\end{array}$ & $\begin{array}{c}\text { Absorción } \\
\mathbf{\%}\end{array}$ & $\begin{array}{c}\text { Humedad } \\
\mathbf{\%}\end{array}$ & $\begin{array}{c}\text { Peso } \\
\text { SSS } \\
\text { Kg }\end{array}$ & $\begin{array}{c}\text { Peso } \\
\text { Hum. } \\
\text { Kg }\end{array}$ \\
\hline Cemento CPC50 & 3,12 & 365,00 & 116,99 & & & 23,72 & 23,72 \\
\hline Agua Potable & 1,00 & 170,00 & 170,00 & & & 11,05 & 10,50 \\
\hline Agua Reciclada & 1,00 & 0,00 & 0,00 & & & 0,000 & 0,000 \\
\hline Arena Fina & 2,60 & 699,80 & 269,15 & 0,36 & 2,07 & 45,49 & 46,26 \\
\hline $\begin{array}{c}\text { Canto Rodado } \\
\text { 06-20 mm }\end{array}$ & 2,63 & 1123,06 & 426,69 & 0,71 & 0,40 & 73,00 & 72,77 \\
\hline $\begin{array}{c}\text { Aditivo Plast. y } \\
\text { Superfluidificante }\end{array}$ & 1,18 & 2,56 & 2,17 & & & 0,17 & 0,17 \\
\hline Aire & 0,0012 & 0,02 & 15,00 & & & 0,001 & 0,001 \\
\hline
\end{tabular}

\subsection{Elaboración del hormigón}

Como se mencionó, el diseño de las mezclas se estableció de acuerdo con las proporciones empleadas por la empresa elaboradora con excepción del reemplazo parcial del agua potable por el agua de lavado en estudio. Por lo tanto, se elaboraron tres dosificaciones con igual cantidad de materiales, pero variando el porcentaje de agua reciclada a incorporar en el orden de $0 \%, 25 \%$ y $35 \%$ respecto al contenido de agua total de la dosificación patrón. En total se elaboraron y evaluaron nueve pastones de $65 \mathrm{dm}^{3}$ de volumen cada uno (Tablas 3). Se replicó tres veces la elaboración para cada porcentaje de reemplazo con el fin de evaluar desvíos o patrones en el comportamiento de la dosificación, para luego enfocar el estudio en los mismos.

Inicialmente, el primer pastón contenía sólo agua potable, recolectada directamente del laboratorio, siendo nombrado como el pastón de referencia. En esta etapa, se agregaron los materiales a la hormigonera y se mantuvieron en el proceso de mezclado durante aproximadamente 5 minutos hasta que la mezcla fuese homogénea. Se procedió al volcado sobre una superficie metálica e inmediatamente se realiza la medición de la temperatura inicial del pastón. Poco después, se llevó a cabo una prueba de asentamiento mediante el tronco cono de Abrams (según Norma IRAM 1536), la determinación de la densidad aparente o peso unitario volumétrico (PUV) y la medición del contenido de aire mediante el aparato de Washington (según Norma IRAM 1602). También se realizó la toma de muestra para la determinación del tiempo de comienzo de fragüe del mortero de hormigón. De manera simultánea se realizó el moldeo de probetas para los ensayos de resistencia a compresión (según Norma IRAM 1524). Posteriormente, el procedimiento la elaboración de los pastones siguió las mismas condiciones y proporciones que el pastón de referencia, pero con el reemplazo parcial del agua de mezclado por el agua en estudio. 
Para la determinación del tiempo de fragüe del mortero de cada pastón se obtuvieron muestras de mortero de cemento, el cual fue tamizado utilizando tamiz de abertura 4,75 $\mathrm{mm}$, para luego moldear sobre probetas cilíndricas de $100 \mathrm{~mm}$ de diámetro y $100 \mathrm{~mm}$ de alto, las que se dejaron en cámara de curado para mantener constantes las condiciones de temperatura y humedad de las muestras. Para las mediciones de resistencia a la penetración se empleó un penetrómetro de hormigón ligero tipo resorte, basado en ASTM C403, mediante el cual se considera que el fraguado inicial se da con un valor de penetración de aproximadamente $35 \mathrm{~kg} / \mathrm{m}^{2}$.

Las probetas cilíndricas de ensayo se moldearon por triplicado y luego se almacenaron en cámara de curado para la determinación de la resistencia a la compresión a las edades de 7, 28 y 65 días. En total, se moldearon 27 probetas, 9 para cada dosificación divididas en las tres edades diferentes. Las muestras se sometieron a al ensayo de compresión estandarizada (según Norma IRAM 1546).

\section{RESULTADOS}

\subsection{Hormigón fresco}

Como se mencionó, los parámetros característicos del hormigón en estado fresco evaluados fueron la consistencia mediante el ensayo del tronco cono de Abrams, temperatura inicial del pastón, PUV y contenido de aire mediante aparato de Washington, Estos resultados se presentan en la Tabla 4.

Tabla 4. Valores de parámetros característicos del hormigón en estado fresco.

\begin{tabular}{|c|c|c|c|c|c|c|c|c|c|}
\hline \multirow{2}{*}{$\begin{array}{c}\text { Agua } \\
\text { Reciclada } \\
\text { (RW) }\end{array}$} & \multirow{2}{*}{$\begin{array}{c}\text { Prueba } \\
\mathrm{N}^{\circ}\end{array}$} & \multicolumn{2}{|c|}{$\begin{array}{c}\text { Asentamiento } \\
{[\mathrm{cm}]}\end{array}$} & \multicolumn{2}{|c|}{$\begin{array}{c}\text { Temperatura } \\
\mathbf{H}^{\circ}\left[{ }^{\circ} \mathbf{C}\right]\end{array}$} & \multicolumn{2}{|c|}{$\begin{array}{c}\text { Contenido de } \\
\text { Aire [\%] }\end{array}$} & \multicolumn{2}{|c|}{ PUV $\left[\mathrm{Kg} / \mathrm{m}^{3}\right]$} \\
\hline & & Valor & Prom. & Valor & Prom. & Valor & Prom. & Valor & Prom. \\
\hline \multirow{3}{*}{ RW $0 \%$} & 1 & 12,50 & \multirow{3}{*}{15,33} & 29,00 & \multirow{3}{*}{25,50} & 2,80 & \multirow{3}{*}{2,37} & 2354,0 & \multirow{3}{*}{2372,00} \\
\hline & 2 & 18,00 & & 25,20 & & 1,80 & & 2388,0 & \\
\hline & 3 & 15,50 & & 22,30 & & 2,50 & & 2374,0 & \\
\hline \multirow{3}{*}{ RW 25\% } & 1 & 17,00 & \multirow{3}{*}{18,33} & 28,70 & \multirow{3}{*}{25,43} & 2,20 & \multirow{3}{*}{2,33} & 2365,0 & \multirow{3}{*}{2324,00} \\
\hline & 2 & 20,00 & & 24,60 & & 2,30 & & 2319,0 & \\
\hline & 3 & 18,00 & & 23,00 & & 2,50 & & 2290,0 & \\
\hline \multirow{3}{*}{ RW 35\% } & 1 & 18,50 & \multirow{3}{*}{21,33} & 29,10 & \multirow{3}{*}{25,97} & 2,30 & \multirow{3}{*}{2,17} & 2388,0 & \multirow{3}{*}{2374,33} \\
\hline & 2 & 24,50 & & 24,50 & & 1,90 & & 2376,0 & \\
\hline & 3 & 21,00 & & 24,30 & & 2,30 & & 2359,0 & \\
\hline
\end{tabular}

La consistencia normal o promedio del pastón realizado mediante la dosificación patrón, es decir sin incorporación de agua reciclada, es del orden de los $15 \mathrm{~cm}$, de acuerdo a los registros informados por la empresa con un desvío del orden de $\pm 3,00 \mathrm{~cm}$, observamos en la Tabla 4 que para todos los casos se obtuvo dicha variación en el asentamiento medido mediante cono de Abrams, con un valor promedio de $15,33 \mathrm{~cm}$.

Luego para la incorporación de un $25 \%$ de agua reciclada respecto al agua total de la dosificación, se observa que los valores de asentamientos obtenidos varían ligeramente respecto a los obtenidos con la dosificación patrón, en el orden de +3 puntos de asentamiento, con un valor promedio de $18,33 \mathrm{~cm}$. 
Para el caso de la incorporación de un $35 \%$ de agua reciclada respecto al agua total de la dosificación, se observa un significativo aumento de la consistencia de los pastones, con un valor promedio de $21,33 \mathrm{~cm}$ respecto a $\operatorname{los} 15,33 \mathrm{~cm}$ de la dosificación patrón, es decir el doble de variación (+ 6 puntos de asentamiento medido) en comparación con la incorporación de un $25 \%$ de agua reciclada. Se observaron pastones de aspecto fluido, homogéneos y uniformes, con una leve exudación y presencia de burbujas de aire. Sin embargo, de acuerdo a los valores obtenidos de aire incorporado no se observaron significativas variaciones, al igual que con la temperatura inicial de elaboración, con un valor promedio del orden de $\operatorname{los} 25,63{ }^{\circ} \mathrm{C}$. El peso unitario volumétrico de los pastones fue del orden de $\operatorname{los} 2356,80 \mathrm{~kg} / \mathrm{m}^{3}$.

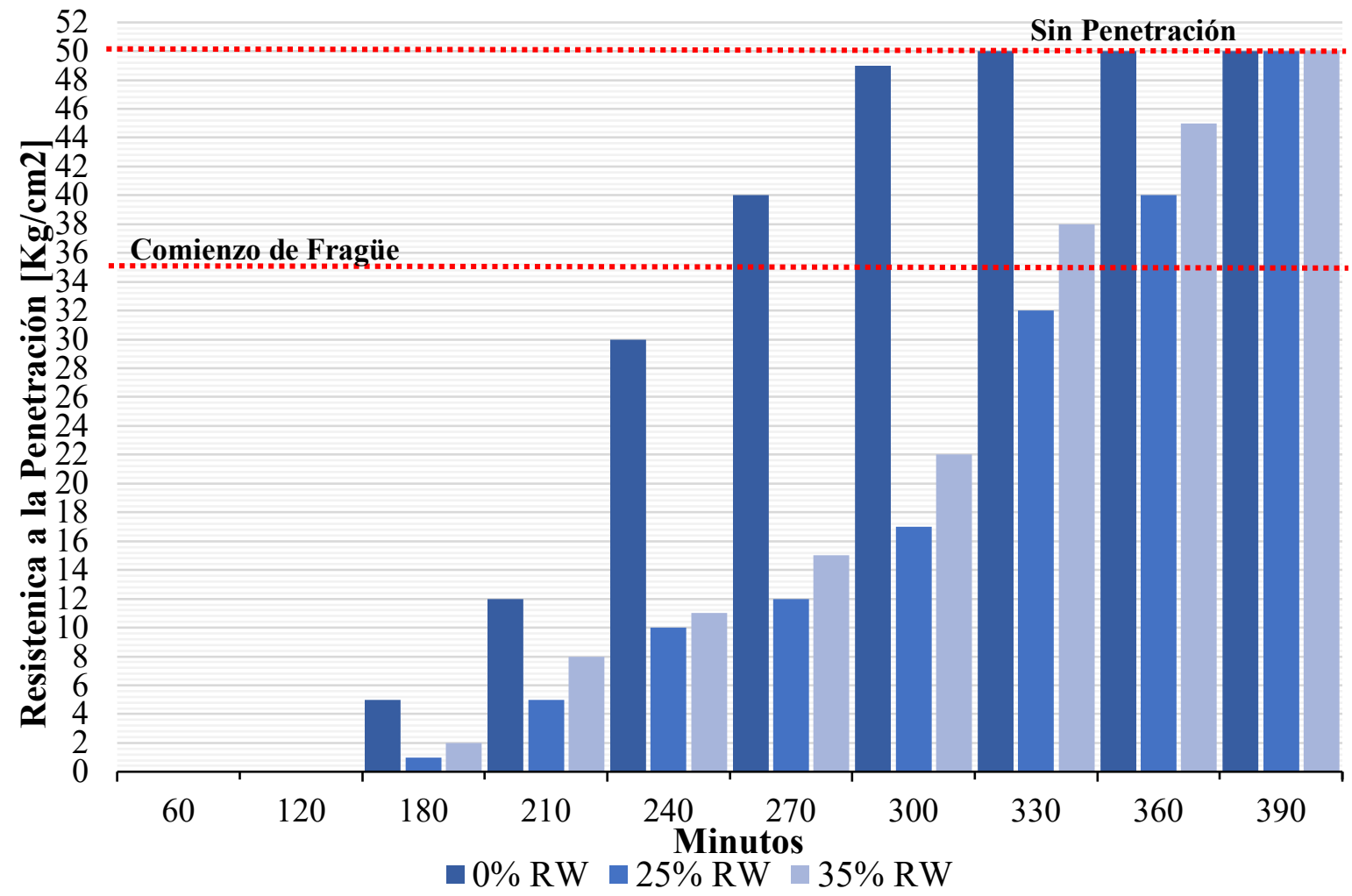

Figura 7. Tiempo de fragüe del hormigón según porcentaje de variación de agua reciclada.

Como se observa en la Figura 7, para la dosificación patrón, el comienzo de fragüe se da de manera acelerada pasados los 240 minutos desde el primer contacto de las partículas de cemento con el agua, alcanzando resistencias superficiales significativas al cabo de los 270 minutos desde el mezclado, comportamiento habitual para este tipo de dosificación. Si se comparan los tiempos de fragüe de los hormigones con incorporación de agua reciclada se observa un patrón de comportamiento para este ensayo, donde el proceso de hidratación se desarrolla de manera paulatina y más lenta, alcanzando el comienzo de fragüe al cabo de 330 minutos en adelante.

A partir de estos resultados podemos concluir que, a medida que se incrementa el porcentaje de reemplazo de agua reciclada, los hormigones presentaron una variación de la consistencia de plástica a fluida evidenciado por el aumento del asentamiento, y un aumento del tiempo de comienzo de fragüe, en referencia a la dosificación base y para los mismos tiempos de prueba.

Cabe aclarar que, en base a la bibliografía consultada, el efecto del incremento de agua reciclada debe ser una reducción de la fluidez debido a la presencia de las partículas sólidas y alta alcalinidad. Esta última genera una influencia en las propiedades eléctricas en la superficie de las partículas de cemento. 
Debido a esto, los resultados obtenidos se podrían justificar con el empleo del aditivo superplastificante, cuya molécula permite el recubrimiento total de las partículas de cemento incorporándoles cargas de signo negativo que neutralizan las fuerzas de atracción electrostáticas existentes entre dichas partículas y dificultan el citado fenómeno de floculación. A su vez se podría considerar que el mayor valor del asentamiento obtenido, con el incremento del contenido de agua reciclada, puede estar también influenciado por rastros de aditivo superfluidificante presentes en el agua de lavado.

\subsection{Hormigón en estado endurecido}

En la Figura 8 se presentan los valores de la resistencia a la compresión simple de los hormigones con $0 \%, 25 \%$ y $35 \%$ de agua reciclada, para las edades de 7, 28 y 56 días. Para el hormigón patrón se obtuvieron valores del orden a los informados por la planta elaboradora, y se observa que para la edad temprana de 7 días las resistencias de los pastones elaborados con agua reciclada también presentan un comportamiento similar. Sin embargo, para edades mayores tales como 28 y 56 días, conforme aumenta el porcentaje de agua reciclada, los valores de resistencia a la compresión simple presentaron una tendencia a la disminución del orden del 10 al $11 \%$.

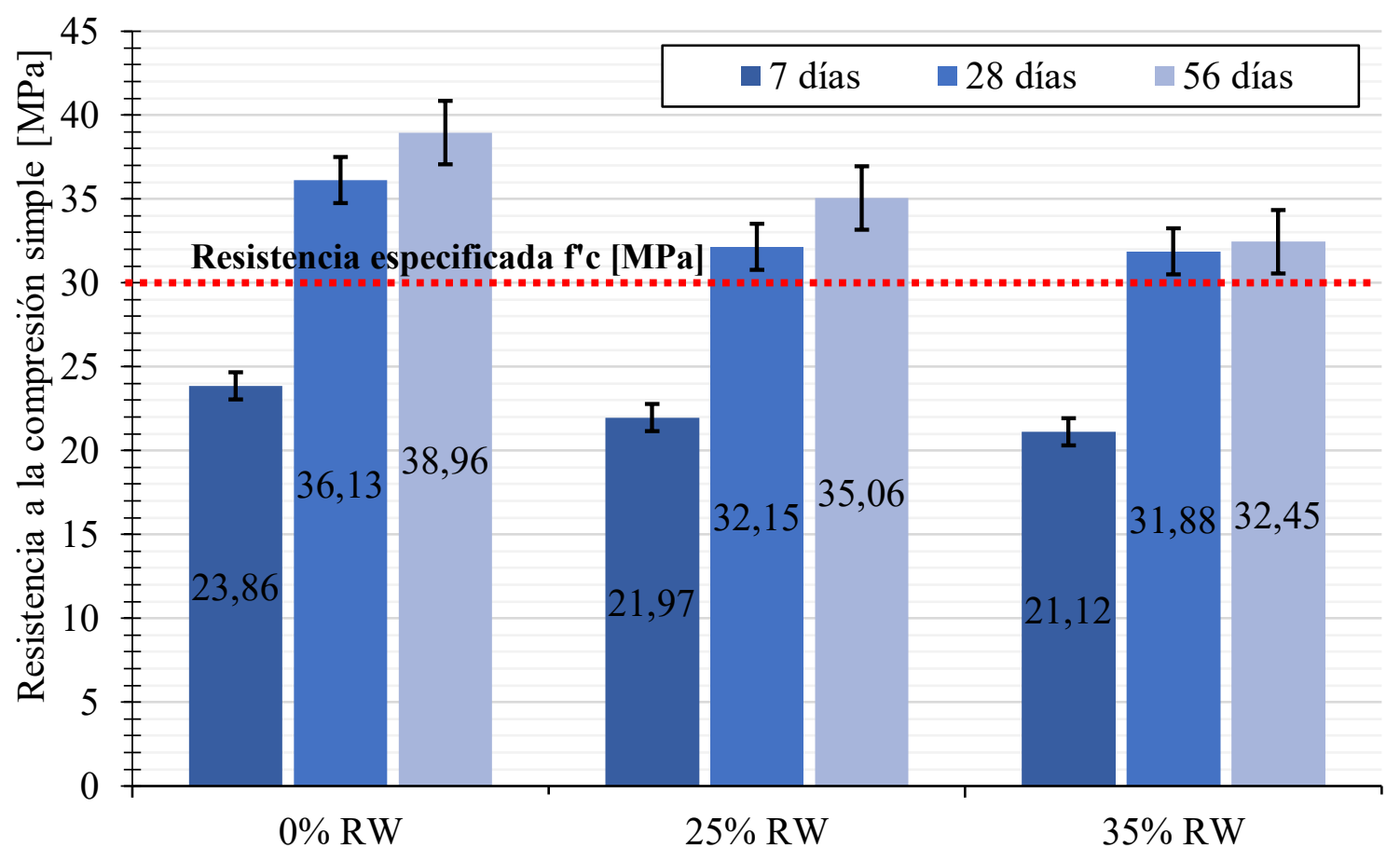

Figura 8. Variación de la resistencia a la compresión simple según porcentaje de agua reciclada por edades.

Al analizar los datos, si bien todas las muestras cumplen con la resistencia especificada considerada por los criterios de conformidad del hormigón bajo MODO I de control, se aprecia la tendencia a una disminución de la resistencia a medida que se incrementa el porcentaje de agua residual.

En principio se puede concluir que, para estas dosis, el agua residual de la empresa se puede utilizar en la elaboración de este tipo de mezclas comerciales, ya que no tiene un comportamiento que impacte negativamente y de forma significativa. 


\section{CONSIDERACIONES FINALES}

El presente trabajo es la primera parte de un proyecto de investigación y desarrollo de tres años de duración. Con él se buscó evaluar el comportamiento de una dosificación patrón o comercial, analizando la variación de sus parámetros característicos en estado fresco y endurecido, ante la incorporación de porcentajes relativos de agua reciclada producto del lavado de camiones mixers. Basado en los resultados obtenidos, se establecieron las siguientes consideraciones finales:

El incremento del contenido de agua reciclada aumentó progresivamente el asentamiento (es decir, aumentó la fluidez de la mezcla) pasando de una consistencia plástica a muy plástica y fluida. Esto se puede deber al elevado $\mathrm{pH}$ que modifica la reología de las mezclas.

El proceso de fragüe se produce de manera más lenta en comparación con la dosificación base. Es decir, el comienzo de fragüe se registra a mayores tiempos a medida que se incrementa el porcentaje de reemplazo de agua reciclada. En principio habría una incidencia de la alta alcalinidad del agua en el retraso del comienzo del fraguado, pero se deben realizar más estudios al respecto.

Las resistencias de los pastones elaborados con agua reciclada presentan un comportamiento similar a la del pastón de referencia para la edad temprana de 7 días. Sin embargo, conforme aumenta el porcentaje de agua reciclada, los valores de resistencia a la compresión simple presentaron una tendencia a la disminución. No obstante, los hormigones que contienen agua reciclada presentaron resistencias a la compresión compatibles con la dosificación de referencia, alcanzando el $88 \%$ para $35 \%$ de reemplazo de agua reciclada y cumpliendo con los requerimientos de la resistencia de diseño.

En conjunto, fue posible producir hormigones con sustitución parcial de agua potable por reciclada, sin deterioros significativos en propiedades frescas y endurecidas. Sin embargo, cabe aclarar que estos son los primeros resultados de la investigación con los agregados y cemento de la zona, y se continuará evaluando el efecto del reemplazo sin el empleo de aditivos químicos en las mezclas con el fin de identificar su influencia en los resultados. Por otro lado, también se buscará evaluar los parámetros de durabilidad de los hormigones.

\section{AGRADECIMIENTOS}

Los autores agradecen a la Universidad Tecnológica Nacional Facultad Regional Concordia, al Grupo de Investigación en Ingeniería, Materiales y Ambiente (GIICMA) por el apoyo a través de la Secretaría de Ciencia, Tecnología y Posgrado. Agradecemos la colaboración de los alumnos becarios Santiago Hernández Solís, Cristian Gómez, Mariano Urribarri y Alejandro Yoly así como al encargado de Laboratorio de Tecnología del Hormigón de la UTN-Concordia. Agradecemos también a la Dra. Natalia Tesón por su colaboración y asistencia en las pruebas de análisis de las muestras de agua. Finalmente agradecemos a la empresa Vecchio SRL por proporcionar las dosificaciones y los materiales necesarios para la elaboración de las mezclas de prueba utilizados en esta investigación.

\section{REFERENCIAS}

Asociación de Fabricantes de Cemento Portland AFCP. (2019). “Anuario. Datos estadísticos año 2019”. http://afcp.info/Anuarios/Anuario-2019.pdf

Asociación Argentina de Hormigón Elaborado AAHE. (2019). "Estadísticas de la Producción de Hormigón Elaborado 2015 al 2019”. https://hormigonelaborado.com/wpcontent/uploads/2020/06/Producci\%C3\%B3n-2019.pdf 
Balzamo, H., Cabrera, O., \& et.al. (2012). “Ese material llamado hormigón”. Buenos Aires, Argentina. ISBN 978-987-21660-5-2.

Instituto Argentino de Normalización y Certificación IRAM (2017). IRAM 50.000. Norma argentina: Cemento. Cementos para uso general. Cuarta edición.

Instituto Argentino de Normalización y Certificación IRAM (1997). IRAM 1.627. Norma argentina: Agregados. Granulometría de los agregados para hormigones. Primera edición.

Instituto Argentino de Normalización y Certificación IRAM (2012). IRAM 1.601. Norma argentina: Agua para morteros y hormigones de cemento. Tercera edición.

Instituto Argentino de Normalización y Certificación IRAM (2020). IRAM 1.536. Norma argentina: Hormigón fresco de cemento. Método de ensayo de la consistencia utilizando el tronco de cono. Segunda edición.

Instituto Argentino de Normalización y Certificación IRAM (1988). IRAM 1.602-1. Norma argentina: Hormigón de cemento pórtland. Método por presión para la determinación del contenido de aire en mezclas frescas de hormigones y morteros - Método A. Primera edición.

Instituto Argentino de Normalización y Certificación IRAM (2015). IRAM 1.524. Norma argentina: Hormigón de cemento. Preparación y curado en obra de probetas para ensayos de compresión y de tracción por compresión diametral. Cuarta edición.

Instituto Argentino de Normalización y Certificación IRAM (2013). IRAM 1.546. Norma argentina: Hormigón de cemento. Método de ensayo de compresión. Tercera edición.

INTI-CIRSOC. (2005). Reglamento Argentino de Estructuras de Hormigón. (INTI, Ed.) Ministerio de Planificación Federal, Inversión Pública y Servicios, Secretaría de Obras Públicas de la Nación. Argentina.

Ms. Ing. Maximiliano Segerer. (2020). "Nueva Norma IRAM de Hormigón Elaborado". Seminario Online de la Industria del Hormigón Elaborado. Asociación Argentina del Hormigón Elaborado (AATH). Buenos Aires, Argentina, pp. 37-39.

Sandrolini, F., \& Franzoni, E. (2001). "Waste wash water recycling in ready-mixed concrete plants". Cement and Concrete Research, 31(3), pp. 485-489. https://doi.org/10.1016/S00088846(00)00468-3

Paulo Ricardo de Matos, Luiz Roberto Prudêncio Jr., Ronaldo Pilar, Philippe Jean Paul Gleize, Fernando Pelisser. (2020). "Use of recycled water from mixer truck wash in concrete: Effect on the hydration, fresh and hardened properties". Construction and Building Materials, 230. doi: ISSN 0950-0618.

ASTM International. (1999). ASTM C 403/C 403M - 99. Método de ensayo. Determinación del tiempo de fraguado de mezclas de concreto por su resistencia a la penetración. http://www.astm.org 